\title{
Classification of Rickettsia tsutsugamushi in a New Genus, Orientia gen. nov., as Orientia tsutsugamushi comb. nov.
}

\author{
AKIRA TAMURA, ${ }^{*}$ NORIO OHASHI, HIROSHI URAKAMI, AND SADAO MIYAMURA \\ Department of Microbiology, Niigata College of Pharmacy, Niigata, Niigata 950-21, Japan
}

\begin{abstract}
Recent studies of Rickettsia tsutsugamushi have demonstrated clearly the phenotypic and genotypic differences between this microorganism and other species belonging to the genus Rickettsia. Therefore, classification of $\boldsymbol{R}$. tsutsugamushi in a new genus, Orientia gen. nov., is proposed.
\end{abstract}

The genus Rickettsia includes etiological agents of human diseases, including typhus, spotted fever, and scrub typhus. All of the microbes belonging to this genus are similar in the following respects: they exhibit obligate intracellular parasitism, they are morphologically similar to gram-negative bacteria, they survive in both vertebrate and arthropod hosts, and human infection is mediated by arthropods. However, recent studies of Rickettsia tsutsugamushi, which is the only species in the scrub typhus group, have revealed some differences between this species and other species belonging to the genus Rickettsia. The most striking difference is in the structure of the outer envelope; as revealed by electron microscopy, the outer leaflet of the cell wall of $R$. tsutsugamushi is considerably thicker than the inner leaflet, while the opposite is true of the other Rickettsia species (36). In addition, chemically, $R$. tsutsugamushi lacks constitutional components of peptidoglycan and lipopolysaccharide, such as muramic acid, glucosamine, hydroxy fatty acids, and 2-keto-3-deoxyoctonic acid, suggesting that neither peptidoglycan nor lipopolysaccharide is present in $R$. tsutsugamushi (4), while these substances are generally found in the other species belonging to the genus Rickettsia (1, $8,15,24,35) . R$. tsutsugamushi is very soft and fragile $(39,44)$, which reflects the lack of peptidoglycan in this microorganism, and the growth of $R$. tsutsugamushi is more resistant to penicillin than the growth of other rickettsiae $(21,32)$.

The protein composition of $R$. tsutsugamushi as determined by sodium dodecyl sulfate-polyacrylamide gel electrophoresis is very different from the protein compositions of the other rickettsiae, especially in the envelopes (43). In $R$. tsutsugamushi, a $54-$ to $58-\mathrm{kDa}$ protein (designated the $56-\mathrm{kDa}$ protein) is most abundant and is located on the cell surface (16, 41). Other major proteins $(80,46,43,39,35,28$, and $25 \mathrm{kDa})$ are also located on the rickettsial surface (41); three of these proteins, the 25-, 28-, and also 56-kDa proteins, are heat modifiable $(16,22,46)$. Another abundant protein, the $60-\mathrm{kDa}$ protein, which is located inside the rickettsial cells, has been shown to exhibit homology to the GroEL family of proteins (37). On the other hand, rickettsiae belonging to the typhus and spotted fever groups resemble each other in their protein compositions. The major antigenic proteins located on the surfaces of these organisms have been estimated to have the molecular sizes of 150 to 180,110 to $130,49,32,27.5$, and 16.5 to $17.5 \mathrm{kDa}(51,53)$. The two proteins larger than $110 \mathrm{kDa}$ are immunodominant antigens and have both heat-labile and heatstable antigenic sites $(5-7,12,15)$. The $16.5-$ to $17.5-\mathrm{kDa}$ antigen is a lipoprotein and exhibits common antigenicity among

\footnotetext{
* Corresponding author. Mailing address: Department of Microbiology, Niigata College of Pharmacy, 5-13-2 Kamishin'ei-cho, Niigata, Niigata 950-21, Japan.
}

the species $(9,10)$. Three proteins that range in size from 20 to $32 \mathrm{kDa}$ and 120 -kDa proteins are heat modifiable $(8,12,13)$.

R. tsutsugamushi can be cultivated in the yolk sacs of developing chicken embryos and can be grown in culture in various cell lines, including the HeLa, BHK, Vero, and L929 cell lines. Most of the microorganisms grow in the perinuclear cytoplasm of host cells, and they release themselves by pushing out the host cytoplasmic membrane from the inside like the budding process observed with enveloped viruses $(49,50)$. The budding rickettsiae accumulate at a high density on the host cell surface. Such budding forms are not commonly seen in the other Rickettsia species. In addition, the electron-lucent halo zones which are commonly observed around growing cells of the other Rickettsia species are not observed with $R$. tsutsugamushi. The doubling time of $R$. tsutsugamushi is about 9 to $18 \mathrm{~h}$ in well-adapted cells $(22,50)$.

Various antigenic variants are recognized among the strains of $R$. tsutsugamushi. The strains of the Gilliam, Karp, and Kato types are well known, but other antigenic types, such as Shimokoshi, Kawasaki, Kuroki and others, have also been described $(14,31,42,55)$. These antigenic types are distinguishable immunologically, especially with strain- or type-specific monoclonal antibodies. The main type-specific antigen is a $56-\mathrm{kDa}$ protein located on the rickettsial cell surface $(17,23$, 41 , although this antigen also has epitopes common among the antigenic variants $(17,26)$. The deduced primary amino acid sequences of this protein from six variants were determined by a genomic sequence analysis $(29,30,38)$, which demonstrated that these molecules exhibit levels of homology ranging from 60 to $82 \%$ between the variants and have four variable domains (30). The 22-, 47-, and $110-\mathrm{kDa}$ proteins also exhibit both common and type-specific antigenicities $(17,26)$. Our recent observations indicate that each antigenic type strain can be further classified into several subtypes on the basis of the results of analyses performed with monoclonal antibodies and restriction fragment length polymorphism analysis of 56-kDa protein genes amplified by a PCR (unpublished data), suggesting that many other variants with various levels of relationship to prototype strains may be found in future studies. Furthermore, differences in virulence for mice have been observed among the strains of $R$. tsutsugamushi $(19,22$, 43); most strains of the Shimokoshi, Kawasaki, and Kuroki types are avirulent in mice. The numerous differences in antigenic and/or genetic structure and in virulence for mice among the strains are unique to $R$. tsutsugamushi.

$R$. tsutsugamushi is transmitted to human and other vertebrate hosts by mites. The microbes in the infected mites are maintained for long periods by transovarial transmission (33, $34,40,45,47,48,54)$. Ticks, lice, and fleas, which serve as vectors or reservoirs of the typhus and spotted fever group rickettsiae, do not participate in $R$. tsutsugamushi transmission. 
In the Weil-Felix reaction, sera of scrub typhus patients agglutinate Proteus mirabilis OXK antigen (3), but not Proteus vulgaris OX19 and OX2 antigens. The antigenic component in $R$. tsutsugamushi shared with OXK antigen has not been identified, although lipopolysaccharide antigens have been shown to be involved in other rickettsiae (2).

In addition to determining the phenotypic differences between $R$. tsutsugamushi and other species belonging to the genus Rickettsia, we recently analyzed the 16S rRNA sequence of $R$. tsutsugamushi and determined the position of this rickettsia on a phylogenetic tree containing 38 species belonging to the Proteobacteria (28). Our results indicated that Gilliam and Kato strains of $R$. tsutsugamushi clustered together at a position that was far from the subcluster containing the other members of genus Rickettsia (Rickettsia prowazekii, Rickettsia typhi, Rickettsia rickettsii, and Rickettsia sibirica). The levels of similarity among $R$. prowazekii, $R$. rickettsii, and $R$. sibirica were more than $98.1 \%$ (evolutionary distances, less than 0.017485 ), but the levels of similarity between these three species and Gilliam and Kato strains of $R$. tsutsugamushi were 90.2 to $90.6 \%$ (evolutionary distances, 0.097480 to 0.106272 ) and the levels of similarity between Gilliam and Kato strains and selected members of the genera Ehrlichia and Bartonella (Rochalimaea) were 81.0 to $82.9 \%$ (evolutionary distances, 0.180613 to 0.207974 ). The levels of similarity among the antigenic variants of $R$. tsutsugamushi, including Gilliam, Kato, Karp, Kawasaki, Kuroki, and Shimokoshi strains, were more than $98.5 \%$ (evolutionary distances, less than 0.01529 ).

Thus, on the basis of 16S rRNA sequences, $R$. tsutsugamushi was found to be distinct from other species belonging to the genus Rickettsia and also to be not specifically related to other members of the order Rickettsiales. In addition, the evolutionary distances between $R$. tsutsugamushi and other species belonging to the genus Rickettsia were nearly equal to the evolutionary distances that separate other genera belonging to the order Rickettsiales. Since a working taxonomy is generally considered from both phenotypic and phylogenetic aspects, the lines of evidence regarding $R$. tsutsugamushi described above may provide sufficient reason to separate $R$. tsutsugamushi from the genus Rickettsia. Therefore, we propose that $R$. tsutsugamushi should be removed from the genus Rickettsia and placed in a new genus that is separated from the genus Rickettsia.

The causative agent of scrub typhus was discovered by several Japanese workers, and the following designations have been proposed for this organism: Theileria tsutsugamushi, a member of the Protozoa, proposed by Hayashi in 1920 (18); Rickettsia orientalis, proposed by Nagayo et al. in 1930 (25); and Rickettsia tsutsugamushi, proposed by Ogata in 1931 (27). Since the publication of Bergey's Manual of Determinative Bacteriology, 6th ed. (10a), the name Rickettsia tsutsugamushi (Hayashi 1920) Ogata 1931 has been accepted. In this paper, we propose a new genus name for this organism, Orientia, as well as the species name Orientia tsutsugamushi, in honor of both Nagayo et al. and Ogata. This nomenclature is appropriate because this microorganism is widely distributed in eastern Asia.

Description of Orientia gen. nov. Orientia (O. ri. en'ti. a. M. L. fem. n. Orientia, the Orient, the area where the organism is widely distributed). Cells are short rods that are 0.5 to $0.8 \mu \mathrm{m}$ in diameter and 1.2 to $3.0 \mu \mathrm{m}$ long. Obligately intracellular parasite. Gram negative. Each cell is surrounded by a very soft cell wall and cell membrane. Muramic acid, glucosamine, 2-keto-3-deoxyoctonic acid, and hydroxy fatty acids are not present in the cell walls, suggesting that the cells lack peptidoglycan and lipopolysaccharide. As determined by electron micros- copy, the outer leaflet of the cell wall is considerably thicker than the inner leaflet. Flagella and endospores are not formed. A slime layer is not recognized. The cells, which can be stained by Giemsa or Gimenez stain, grow in the yolk sacs of developing chicken embryos and in various cultured cell lines. The microbes are taken into the host cells by phagocytosis, enter the cytoplasm, and grow mainly in the perinuclear cytoplasm. There is no electron-lucent halo zone around the microbes growing in host cells. The microbes are released from the host cells by being covered with the host cell membrane, like the budding form of enveloped viruses. The doubling time is about 9 to $18 \mathrm{~h}$. Very resistant to penicillin. The most abundant constitutional protein has a molecular mass of 54 to $58 \mathrm{kDa}$. This protein and $25-$ and $28-\mathrm{kDa}$ proteins, all of which are located on the cell surface, are heat modifiable. Another abundant protein, the $60-\mathrm{kDa}$ protein, is located inside cells and belongs to the GroEL protein family. Sequence analyses of the $16 \mathrm{~S}$ rRNA reveal that the members of this genus form a cluster on phylogenetic trees and that the levels of similarity are more than $98.5 \%$ (evolutionary distances, less than 0.015129 ). The $\mathrm{G}+\mathrm{C}$ contents are 28.1 to $30.5 \mathrm{~mol} \%$ (as determined by highperformance liquid chromatography) (20).

The members of this genus are classified in one species at the present time, but they exhibit antigenic variety and differ in their virulence for mice. These organisms are found only in the eastern hemisphere. Mites, including several species belonging to the genus Leptotrombidium, are vectors for transmission of the microbes to humans and other vertebrate hosts. In these mites, the microorganisms are transmitted transovarially from female adult mites to their progeny. Sera of patients infected with these microbes agglutinate OXK antigen in the Weil-Felix test.

The genus Orientia belongs to the family Rickettsiaceae together with the genus Rickettsia. On the basis of the results of recent $16 \mathrm{~S}$ rRNA sequences analyses $(11,28)$, retention of the tribe Rickettsieae may not be required in this family. The type species is Orientia tsutsugamushi comb. nov. (basonym, Theileria tsutsugamushi Hayashi [18]).

Description of Orientia tsutsugamushi comb. nov. Only one species, Orientia tsutsugamushi, belongs in the genus Orientia, and characteristics of this species are described above. The description given by Weiss and Moulder (52) can be used as a supplemental description. The type strain is strain Karp (= ATCC VR 150).

We thank Yuzo Yamada (Faculty of Agriculture, Shizuoka University) for his kind advice.

This study was supported in part by grants-in-aid from the Ministry of Education, Science, and Culture, Japan, to A. Tamura (03454185, 06670307), to N. Ohashi $(05770198,06770209)$, and to H. Urakami (06670275).

\section{REFERENCES}

1. Allison, A., and H. Perkins. 1960. Presence of cell walls like those of bacteria in rickettsiae. Nature (London) 188:796-798.

2. Amano, K., M. Fujita, and T. Suto. 1993. Chemical properties of lipopolysaccharide from spotted fever group rickettsiae and their common antigenicity with lipopolysaccharides from Proteus species. Infect. Immun. 61:43504355 .

3. Amano, K., N. Suzuki, M. Fujita, Y. Nakamura, and T. Suto. 1993. Serolog ical reactivity of sera from scrub typhus patients against Weil-Felix test antigens. Microbiol. Immunol. 37:927-933.

4. Amano, K., A. Tamura, N. Ohashi, H. Urakami, S. Kaya, and K. Fukushi. 1987. Deficiency of peptidoglycan and lipopolysaccharide components in Rickettsia tsutsugamushi. Infect. Immun. 55:2290-2292.

5. Anacker, R. L., R. H. List, R. E. Mann, S. F. Hayes, and L. A. Thomas. 1985 Characterization of monoclonal antibodies protecting mice against Rickettsia rickettsii. J. Infect. Dis. 151:1052-1060.

6. Anacker, R. L., R. H. List, R. E. Mann, and D. L. Wiedbrauk. 1986. Antigenic heterogeneity in high- and low-virulence strains of Rickettsia rickettsii 
revealed by monoclonal antibodies. Infect. Immun. 51:653-660.

7. Anacker, R. L., R. N. Philip, E. Casper, W. J. Todd, R. E. Mann, M. R. Johnston, and C. J. Nauck. 1983. Biological properties of rabbit antibodies to a surface antigen of Rickettsia rickettsii. Infect. Immun. 40:292-298.

8. Anacker, R. L., R. N. Philip, J. C. Williams, R. H. List, and R. E. Mann. 1984. Biochemical and immunochemical analysis of Rickettsia rickettsii strains of various degrees of virulence. Infect. Immun. 44:559-564.

9. Anderson, B. E., B. R. Baumstark, and W. J. Bellini. 1988. Expression of the gene encoding the 17-kilodalton antigen from Rickettsia rickettsii: transcription and posttranslational modification. J. Bacteriol. 170:4493-4500.

10. Anderson, B. E., and T. Tzianabos. 1989. Comparative sequence analysis of a genus-common rickettsial antigen gene. J. Bacteriol. 171:5199-5201.

10a.Bengtson, I. A. 1948. Family Rickettsiaceae, p. 1083-1099. In R. S. Breed, E. G. D. Murray, and A. P. Hitchens (ed.), Bergey's manual of determinative bacteriology, 6th ed. The Williams \& Wilkins Co., Baltimore.

11. Brenner, D. J., S. P. O'Connor, H. H. Winkler, and A. G. Steigerwalt. 1993. Proposals to unify the genera Bartonella and Rochalimaea, with descriptions of Bartonella quintana comb. nov., Bartonella vinsonii comb. nov, Bartonella henselae comb. nov., and Bartonella elizabethae comb. nov., and to remove the family Bartonellaceae from the order Rickettsiales. Int. J. Syst. Bacteriol. 43:777-786.

12. Ching, W. M., G. A. Dasch, M. Carl, and M. E. Dobson. 1990. Structural analysis of the $120-\mathrm{kDa}$ serotype protein antigens of typhus group rickettsiae. Ann. N. Y. Acad. Sci. 590:334-351.

13. Dasch, G. A., J. P. Burans, M. E. Dobson, R. I. Jaffe, and W. G. Sewell. 1985. Distinctive properties of components of the cell envelopes of typhus group rickettsiae, p. 54-61. In J. Kazar (ed.), Rickettsiae and rickettsial diseases. Publishing House of the Slovak Academy of Sciences, Bratislava, Czechoslovakia.

14. Elisberg, B. L., J. M. Campbell, and F. M. Bozeman. 1968. Antigenic diversity of Rickettsia tsutsugamushi: epidemiologic and ecologic significance. J. Hyg. Epidemiol. Microbiol. Immunol. 12:18-25.

15. Feng, H. M., D. H. Walker, and J. G. Wang. 1987. Analysis of T-celldependent and -independent antigens of Rickettsia conorii with monoclonal antibodies. Infect. Immun. 55:7-15.

16. Hanson, B. 1984. Characterization of Rickettsia tsutsugamuhsi outer membrane proteins, p. 240-243. In L. Leive and D. Schlessinger (ed.), Microbiology-1984. American Society for Microbiology, Washington, D.C.

17. Hanson, B. 1985. Identification and partial characterization of Rickettsia tsutsugamushi major protein immunogens. Infect. Immun. 50:603-609.

18. Hayashi, N. 1920. Etiology of tsutsugamushi disease. J. Parasitol. 7:53-68.

19. Jerrells, T. R. 1988. Mechanisms of immunity to Rickettsia species and Coxiella burnetii, p. 79-100. In D. H. Walker (ed.), Biology of rickettsial diseases, vol. 2. CRC Press, Boca Raton, Fla.

20. Kumura, K., Y. Minamishima, S. Yamamoto, N. Ohashi, and A. Tamura. 1991. DNA base composition of Rickettsia tsutsugamushi determined by reverse-phase high-performance liquid chromatography. Int. J. Syst. Bacteriôl. 41:247-248.

21. Miyamura, S., T. Ohta, and A. Tamura. 1989. Comparison of in vitro susceptibility of Rickettsia prowazekii, $R$. rickettsii, $R$. sibirica and $R$. tsutsugamushi to antimicrobial agents. Jpn. J. Bacteriol. 44:717-721.

22. Moree, M. F., and B. Hanson. 1992. Growth characteristics and proteins of plaque-purified strains of Rickettsia tsutsugamushi. Infect. Immun. 60:34053415 .

23. Murata, M., Y. Yoshida, M. Osono, N. Ohashi, M. Oyanagi, H. Urakami, A. Tamura, S. Nogami, H. Tanaka, and A. Kawamura, Jr. 1986. Production and characterization of monoclonal strain-specific antibodies against prototype strains of Rickettsia tsutsugamushi. Microbiol. Immunol. 30:599-610.

24. Myers, W., R. Ormsbee, J. Osterman, and C. Wisseman. 1967. The presence of diaminopimelic acid in the rickettsiae. Proc. Soc. Exp. Biol. Med. 125: $459-462$.

25. Nagayo, M., T. Tamiya, T. Mitamura, and K. Sato. 1930. On the virus of tsutsugamushi disease and its demonstration by a new method. Jpn. J. Exp. Med. 8:309-318

26. Oaks, E. V., R. M. Rice, D. J. Kelly, and C. K. Stover. 1989. Antigenic and genetic relatedness of eight Rickettsia tsutsugamushi antigens. Infect. Immun. 57:3116-3122.

27. Ogata, N. 1931. Aetiologie der tsutsugamushi-Kraukheit: Rickettsia tsutsugamushi. Zentralbl. Bakteriol. Parasitenkd. Infektionskr. Hyg. Abt. 1 Orig. 122:249-253.

28. Ohashi, N., M. Fukuhara, M. Shimada, and A. Tamura. 1995. Phylogenetic position of Rickettsia tsutsugamushi and relationship among its antigenic variants by analyses of $16 \mathrm{~S}$ rRNA gene sequences. FEMS Microbiol. Lett. 125:299-304.

29. Ohashi, N., H. Nashimoto, H. Ikeda, and A. Tamura. 1990. Cloning and sequencing of the gene ( $t$ sg 56 ) encoding a type-specific antigen from Rickettsia tsutsugamushi. Gene 91:119-122.

30. Ohashi, N., H. Nashimoto, H. Ikeda, and A. Tamura. 1992. Diversity of immunodominant 56-kDa type-specific antigen (TSA) of Rickettsia tsutsugamushi: sequence and comparative analyses of the genes encoding TSA homologues from four antigenic variants. J. Biol. Chem. 267:12728-12735.
31. Ohashi, N., A. Tamura, H. Sakurai, and S. Yamamoto. 1990. Characterization of a new antigenic type, Kuroki, of Rickettsia tsutsugamushi isolated from a patient in Japan. J. Clin. Microbiol. 28:2111-2113.

32. Raoult, D., and M. Drancourt. 1991. Antimicrobial therapy of rickettsial diseases. Antimicrob. Agents Chemother. 35:2457-2462.

33. Roberts, L. W., G. Rapmund, and F. C. Cadigan, Jr. 1977. Sex ratios in Rickettsia tsutsugamushi-infected and noninfected colonies of Leptotrombidium (Acari: Trombiculidae). J. Med. Entomol. 14:89-92.

34. Roberts, L. W., and D. M. Robinson. 1977. Efficiency of transovarial transmission of Rickettsia tsutsugamushi in Leptotrombidium arenicola (Acari: Trombiculidae). J. Med. Entomol. 13:493-496.

35. Schramek, S., R. Brezina, and J. Kazar. 1977. Some biological properties of an endotoxic lipopolysaccharide from the typhus group rickettsiae. Acta Virol. 21:493-441.

36. Silverman, D., and C. Wisseman. 1978. Comparative ultrastructural study on the cell envelopes of Rickettsia prowazekii, Rickettsia rickettsii and Rickettsia tsutsugamushi. Infect. Immun. 21:1020-1023.

37. Stover, C., D. Marana, G. Dasch, and E. Oaks. 1990. Molecular cloning and sequence analysis of the Sta58 major antigen gene of Rickettsia tsutsugamushi: sequence homology and antigenic comparison of Sta58 to the 60kilodalton family of stress proteins. Infect. Immun. 58:1360-1368.

38. Stover, C. K., D. P. Marana, J. M. Carter, B. A. Roe, E. Mardis, and E. V. Oaks. 1990. The 56-kilodalton major protein antigen of Rickettsia tsutsugamushi: molecular cloning and sequence analysis of the sta56 gene and precise identification of a strain-specific epitope. Infect. Immun. 58:2076-2084.

39. Takahashi, K., H. Urakami, and A. Tamura. 1985. Purification of cell envelopes of Rickettsia tsutsugamushi. Microbiol. Immunol. 29:475-478.

40. Takahashi, M., M. Murata, S. Nogami, E. Hori, A. Kawamura, Jr., and H. Tanaka. 1988. Transovarial transmission of Rickettsia tsutsugamushi in Leptotrombidium pallidum successively reared in the laboratory. Jpn. J. Exp. Med. 58:213-218.

41. Tamura, A., N. Ohashi, H. Urakami, K. Takahashi, and M. Oyanagi. 1985. Analysis of polypeptide composition and antigenic components of Rickettsia tsutsugamushi by polyacrylamide gel electrophoresis and immunoblotting. Infect. Immun. 43:671-675.

42. Tamura, A., K. Takahashi, T. Tsuruhara, H. Urakami, S. Miyamura, H. Sekikawa, M. Kenmotsu, M. Shibata, S. Abe, and H. Nezu. 1984. Isolation of Rickettsia tsutsugamushi antigenically different from Kato, Karp, and Gilliam strains from patients. Microbiol. Immunol. 28:873-882.

43. Tamura, A., H. Urakami, and N. Ohashi. 1991. A comparative view of Rickettsia tsutsugamushi and the other groups of rickettsiae. Eur. J. Epidemiol. 7:259-269.

44. Tamura, A., H. Urakami, and T. Tsuruhara. 1982. Purification of Rickettsia tsutsugamushi by Percoll density gradient centrifugation. Microbiol. Immunol. 26:321-328.

45. Traub, R., and C. L. Wisseman, Jr, 1974. The ecology of chigger-borne rickettsiosis (scrub typhus). J. Med. Entomol. 11:237-303.

46. Urakami, H., N. Ohashi, T. Tsuruhara, and A. Tamura. 1986. Characterization of polypeptides in Rickettsia tsutsugamushi: effect of preparative conditions on migration of polypeptides in polyacrylamide gel electrophoresis. Infect. Immun. 51:948-952

47. Urakami, H., M. Takahashi, E. Hori, and A. Tamura. 1994. An electron microscopic study of vertical transmission of Rickettsia tsutsugamushi in the oogenesis and the spermatogenesis in Leptotrombidium pallidum. Am. J. Trop. Med. Hyg. 50:219-228.

48. Urakami, H., M. Takahashi, M. Murata, and A. Tamura. 1994. Electron microscopic study of the distribution and the vertical transmission of Rickettsia tsutsugamushi in Leptotrombidium pallidum. Jpn. J. Med. Sci. Biol. 47:127-139.

49. Urakami, H., T. Tsuruhara, and A. Tamura. 1982. Penetration of Rickettsia tsutsugamushi into cultured mouse fibroblasts (L cells): an electron microscopic observation. Microbiol. Immunol. 27:251-263.

50. Urakami, H., T. Tsuruhara, and A. Tamura. 1984. Electron microscopic studies of intracellular multiplication of Rickettsia tsutsugamushi in L cells. Microbiol. Immunol. 28:1191-1201.

51. Weiss, E., M. E. Dobson, and G. A. Dasch. 1987. Biochemistry of rickettsiae: recent advances. Acta Virol. 31:271-286.

52. Weiss, E., and J. W. Moulder. 1984. Family I. Rickettsiaceae Pinkerton 1936, $186^{\mathrm{AL}}, 486^{\mathrm{AL}}$, p. $687-698$. In N. R. Krieg and J. G. Holt (ed.), Bergey's manual of systematic bacteriology, vol. 1. Williams \& Wilkins Co., Baltimore.

53. Winkler, H. H. 1990. Rickettsia species (as organisms). Annu. Rev. Microbiol. 44:131-153.

54. Wright, J. D., M. W. Hastriter, and D. M. Robinson. 1984. Observations on the ultrastructure and distribution of Rickettsia tsutsugamushi in naturally infected Leptotrombidium (Leptotrombidium) arenicola (Acari: Trombiculidae). J. Med. Entomol. 21:17-27.

55. Yamamoto, S., N. Kawabata, A. Tamura, H. Urakami, N. Ohashi, M. Murata, Y. Yoshida, and A. Kawamura, Jr. 1986. Immunological properties of Rickettsia tsutsugamushi, Kawasaki strain, isolated from a patient in Kyushu. Microbiol. Immunol. 30:611-620. 